\title{
Papillary Carcinoma Arising from Ectopic Thyroid Tissue in the Spinal Cord
}

\author{
Evan Paulo D. Consencino, Sherry Evett Yap, Cherrie Gail Lumapas-Gonzalez \\ Section of Endocrinology, Diabetes and Metabolism, Department of Internal Medicine, \\ St. Luke's Medical Center, Quezon City, Philippines
}

\begin{abstract}
Ectopically located thyroid tissue is uncommon and if present, is usually found in the lateral neck. Rarely, this may give rise to a carcinoma. The diagnosis rests on post-surgical histopathologic examination. We present a rare case of papillary carcinoma arising from ectopic thyroid tissue in the spinal cord in a 12-year old girl who developed spinal cord compression T4 level in 2004. She underwent laminectomy with tumor excision and histopathologic diagnosis was ependymoma. The spinal cord mass recurred when she was 17 years old (in 2009) and the tumor was excised. Tissue staining with thyroglobulin, thyroid transcription factor-1 (TTF-1) and epithelial membrane antigen (EMA) were positive. Pathologic diagnosis was papillary carcinoma. Subsequently, she underwent a total thyroidectomy to confirm an occult primary thyroid cancer; however, histopathologic examination of the thyroid was benign. Whole body scan using I-131 did not reveal extrathyroidal uptake. Radioactive iodine ablation was not done. She was maintained on a suppressive dose of levothyroxine. She remained paraplegic, with no evidence of tumor recurrence two years since her last surgery.

Keywords: papillary thyroid carcinoma, spinal cord compression, ectopic thyroid
\end{abstract}

\section{Introduction}

Thyroid tissue in an ectopic location is rare, occurring in 1 out of 100,000 to 300,000 persons; and is usually found in the lateral neck. ${ }^{1}$ Ectopic thyroid tissue developing axially is even more rare, with up to $90 \%$ of cases being lingual thyroid tissue arising embryologically from a median anlage from the pharyngeal floor. ${ }^{1,2}$ Very rarely, ectopic thyroid tissue may give rise to a carcinoma. ${ }^{3}$ Carcinogenesis of ectopic thyroid tissue located in midline structures such as lingual thyroid and thyroglossal duct cysts, have a reported incidence of approximately $1 \%$, and usually occurs during the third decade of life. ${ }^{4}$ Almost all cases are diagnosed post-surgically on histopathologic examination. Management of these cases is individualized.

\section{Presentation}

A 12-year old girl developed progressive bilateral lower extremity weakness and sensory deficit, difficulty in ambulation, and bowel and bladder incontinence in 2004. She had no known exposure to ionizing radiation. Maternal and early pediatric histories were unremarkable. She had no family history of malignancy and thyroid disease. Physical examination revealed a lean build; with vital signs, height and weight appropriate for age. The thyroid gland was not enlarged. Chest and abdominal examination were unremarkable. She had full and equal pulses without peripheral edema. Neuromuscular examination revealed decreased manual muscle strength on the lower extremities, hypoesthesia from T4 dermatomal level and hyperreflexia on both lower extremities. Magnetic resonance imaging (MRI) of the thoracolumbar spine revealed a well-defined enhancing nodule in the spinal cord at the level of T3-T4. The nodule measured $1.16 \mathrm{~cm} \times 1.26 \mathrm{~cm} \times 1.58 \mathrm{~cm}$, with intermediate signal intensity in both T1- and T2-weighted studies, with associated edema above and below the lesion from level T2 down to T8-T9 (Figure 1). She underwent a T3-T4 laminectomy with tumor excision 3 months after initial consult (January 2005). Histopathologic exam revealed a $3.0 \mathrm{~cm} \times 1.5 \mathrm{~cm} \times 0.8 \mathrm{~cm}$ mass, microscopically composed of bland cuboidal cells with uniform ovoid nuclei and adequate amphophilic cytoplasm arranged in pseudorosettes, with some cells exhibiting pale-staining to grayish cytoplasm and rare mitotic figures (Figure 2). Immunohistochemical staining for cytokeratin and neuron-specific enolase were positive. Ependymoma was considered in the histopathologic report of the excised mass. Two months postoperatively, the patient had gradual improvement of lower extremity weakness and was able to ambulate by herself. She was then lost to follow up.
ISSN 0857-1074

Copyright (C) 2011 by the JAFES

Received August 22, 2011. Accepted October 5, 2011.
Corresponding author: Evan Paulo D. Consencino, MD.

Diabetes, Thyroid and Endocrine Center

$12^{\text {th }}$ Floor Cathedral Heights Building Complex

St. Luke's Medical Center

E. Rodriguez Avenue, Quezon City, Philippines

Tel. No: 723-0101 local 5210

Fax No.: 723-0101 local 5210 
However, in March 2009, the patient developed gradual progressive weakness of both lower extremities, leading to paralysis. A repeat MRI of the thoracic spine showed an avidly enhancing intramedullary nodule at T3-T4 level, appearing bilobed with irregular margins, measuring 1.13 $\mathrm{cm} \times 1.65 \mathrm{~cm} \times 1.63 \mathrm{~cm}$. The nodule was slightly hyperintense in T1-weighted study (Figure 3) and hypointense on T2-weighted imaging, with some extension to the neural canal at the level of T3-T4. A second laminectomy with tumor excision was done in May 2009. Histopathologic examination of the excised $2 \mathrm{~cm} x$ $1.5 \mathrm{~cm} \times 0.5 \mathrm{~cm}$ mass revealed colloid material within the lumen of follicles or ducts (Figure 4) with papillary architecture and nuclear features consistent with papillary carcinoma. These findings were not seen in the histopathologic examination of the first surgical specimen. Microscopic sections from the second surgical specimen stained positively for thyroglobulin, thyroid transcription factor-1 (TTF-1) and epithelial membrane antigen (EMA) (Figures 5 to 7). The pathologic diagnosis of papillary carcinoma, suggestive of a thyroid primary, supersedes the previous histopathologic diagnosis. Thyroid ultrasound was normal. Thyroid function testing was also normal: thyroid stimulating hormone (TSH) was 1.03 $\mu \mathrm{IU} / \mathrm{mL}$ (normal value 0.35 to 4.94 ), total thyroxine was 9 $\mu \mathrm{g} / \mathrm{dL}$ (normal value 4.9 to 11.7 ), and total triiodothyronine was $1.24 \mathrm{ng} / \mathrm{mL}$ (normal value 0.58 to 1.59). Metastatic workup including CT scans of the neck, chest and abdomen did not reveal any metastatic foci.

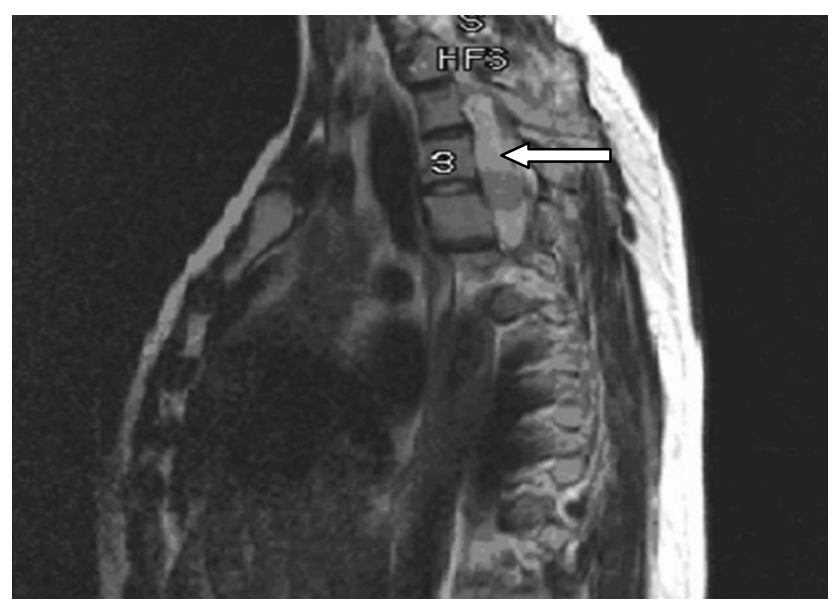

Figure 1. MRI of the thoracolumbar spine, T1 weighted image taken in 2004, showing a well-defined enhancing nodule in the spinal cord at the level of T3-T4 with intermediate signal intensity.

Papillary carcinoma of the thyroid with spinal cord metastasis was the foremost consideration. A total thyroidectomy was performed in July 2009, to confirm occult primary thyroid cancer and to facilitate ablation of residual thyroid tissue for subsequent surveillance for recurrence using radioactive iodine. Histopathologic examination of the thyroid gland revealed nodular hyperplasia after thorough sampling of the entire specimen. Two months after total thyroidectomy, whole body scan using $2 \mathrm{mCi}$ Iodine-131 revealed functioning thyroid remnants in the anterior neck without undue tracer deposition seen elsewhere. Her postoperative stimulated thyroglobulin level was less than $2 \mathrm{ng} / \mathrm{mL}$. She was placed on daily levothyroxine suppressive doses with regular monitoring of thyroid function tests and thyroglobulin level. Radioactive iodine ablation was not indicated since there was no evidence of remaining iodineavid lesions in the spinal cord. She was placed on physical therapy and rehabilitation program after spinal surgery and thyroidectomy. The patient, now 19 years of age, remains paraplegic, with no evidence of active malignancy both clinically and on imaging studies, two years since her last surgery. Thyrotropin levels are adequately suppressed and serial results of thyroglobulin levels are undetectable.

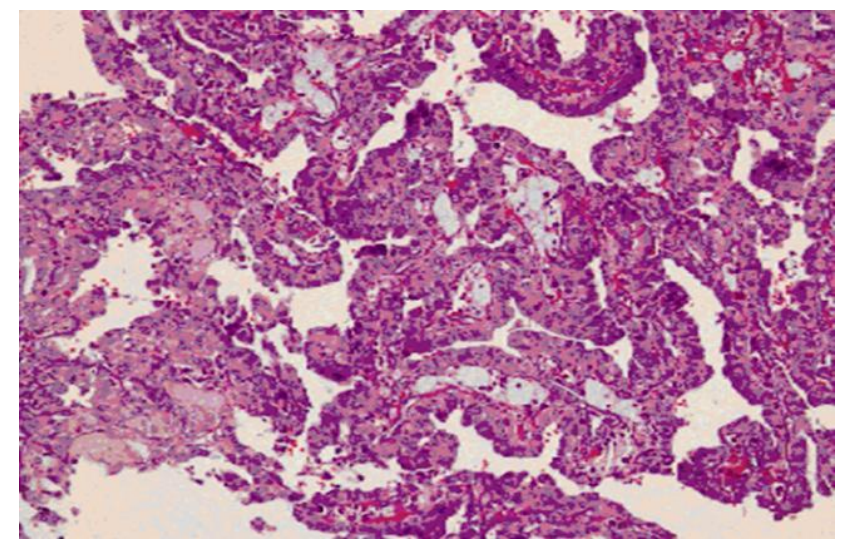

Figure 2. Microscopic examination of the excised spinal cord tumor in 2005 showing bland cuboidal cells with uniform ovoid nuclei and adequate amphophilic cytoplasm, arranged in pseudorosettes; some exhibiting pale-staining to grayish cytoplasm, and rare mitotic figures (H\&E, x40).

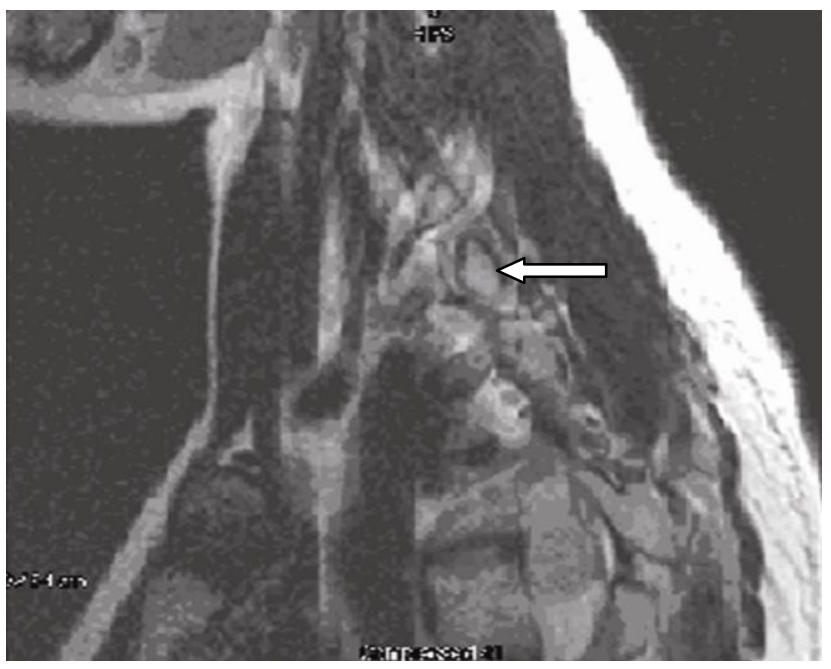

Figure 3. MRI of the thoracic spine T1-weighted image taken in 2009 showing an avidly enhancing intramedullary nodule at T3-T4 level, appearing bilobed with irregular margins, with some extension to the neural canal. 


\section{Discussion}

Thyroid tissue that is located elsewhere from its expected location anterior to the second to fourth tracheal cartilages is ectopic ${ }^{5}$. Embryologically, the thyroid gland develops from a median anlage and a pair of lateral anlages. The embryologic pharyngeal floor gives rise to the median anlage, whereas the fourth and fifth branchial pouches give rise to the lateral anlage. Its descent follows the heart and great vessels and moves caudally from its origin to its location in the neck in front of the trachea. ${ }^{6}$ Aberrant caudal descent of the median anlage during development may give rise to an intrathoracic location of ectopic thyroid tissue. There have been reports of ectopic thyroid tissue occurring in the right ventricle of the heart, ${ }^{7}$ aberrant right carotid thyroid tissue, ${ }^{8}$ carotid bifurcation, ${ }^{5}$ lingual ectopic thyroid, ${ }^{9}$ intrathoracic ectopic thyroid, ${ }^{10}$ substernal goiter, ${ }^{11}$ intralaryngotracheal thyroid ${ }^{12}$ and spinal cord ${ }^{6}$. Carcinomas arising from ectopic thyroid tissue are uncommon. They have been reported to arise from thyroid tissue in thyroglossal duct cysts, lateral aberrant thyroid tissue, lingual thyroid and mediastinal and struma ovarii. ${ }^{2,13}$ Most tumors in the ectopic locations have been papillary carcinomas, mixed follicular and papillary carcinomas or Hürthle cell tumors. ${ }^{14,15}$ However, a carcinoma arising from spinal ectopic thyroid tissue has never been reported in literature.

Differentiating between a metastatic thyroid carcinoma and malignant transformation of an ectopic thyroid tissue is difficult and can only be done after surgery, as in this case. There are no clinical, biochemical, or imaging parameters that may assist in determining the nature of the lesion; and histological examination is always required for definitive diagnosis. ${ }^{15}$ True ectopic thyroid tissue has an arterial supply independent of the cervical arteries that supply the thyroid; the cervical thyroid gland is normal or absent with no history of surgery; the cervical thyroid gland does not have a similar pathologic process as the ectopic tumor, and there is no history or evidence of thyroid malignancy. ${ }^{16}$

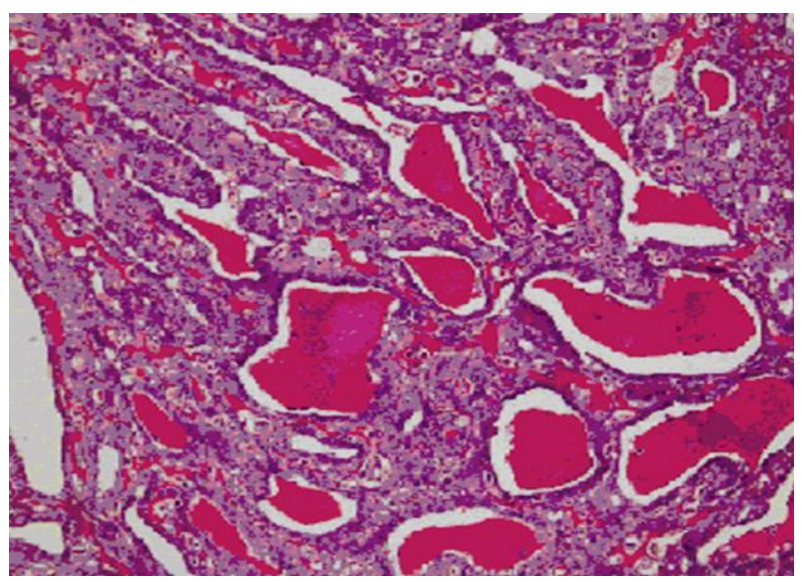

Figure 4. Microscopic exam of excised spinal cord tumor in 2009, showing colloid material within the lumen of follicles, papillary architecture and nuclear features consistent with papillary carcinoma (H\&E, x40).

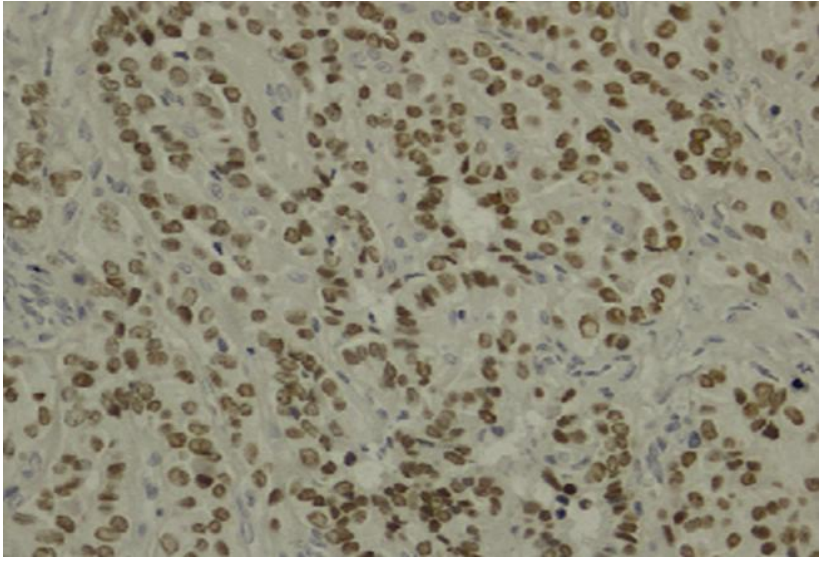

Figure 5. Microscopic exam of excised spinal cord tumor in 2009 (TTF-1, x400).

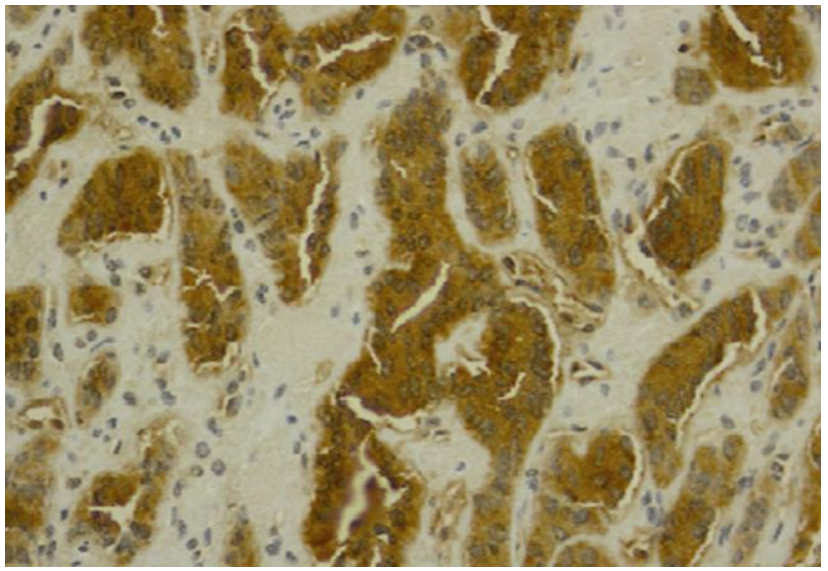

Figure 6. Microscopic exam of excised spinal cord tumor in 2009 (Thyroglobulin, x400).

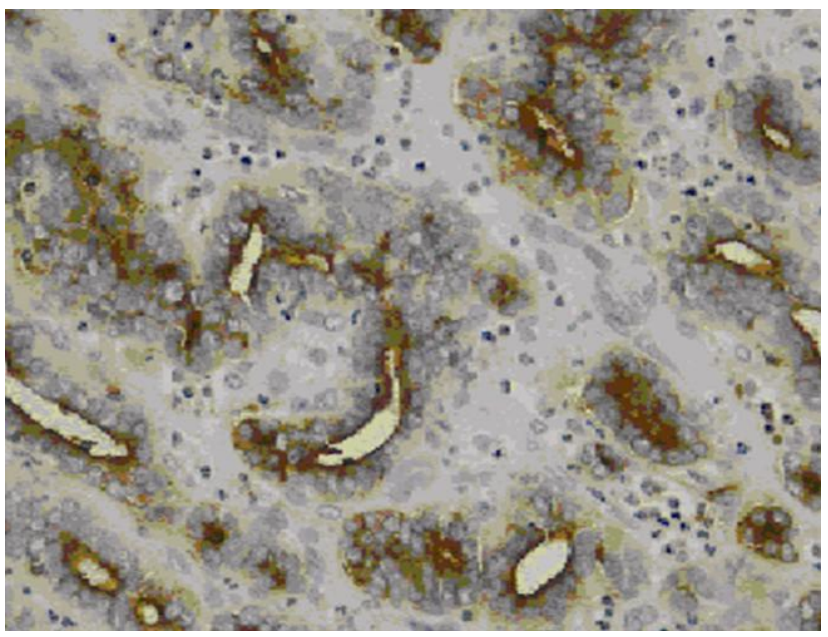

Figure 7. Microscopic exam of excised spinal cord tumor in 2009 (EMA, x400).

Although a metastatic papillary thyroid cancer was initially considered, the migration of papillary carcinoma from a primary thyroid to distant sites bypassing cervical lymph nodes is unusual. Also, a review of the postthyroidectomy histopathology did not reveal malignancy in the thyroid, leading us to conclude that the tumor 
excised from the patient's spinal cord was ectopic thyroid tissue that transformed into papillary carcinoma. The postoperative whole body scan that was negative for iodine avid lesions outside the thyroid bed may reflect either complete resection of tumor in the spinal cord or poor iodine avidity. She did not receive high dose radioiodine ablation, and was given a suppressive levothyroxine dose at $100 \mathrm{mcg}$ daily to prevent recurrence.

\section{Conclusion}

Metastasis from a primary thyroid carcinoma must first be ruled out before considering malignant transformation of an ectopic thyroid tissue, which is a rare occurrence. There are no clinical, biochemical, or imaging parameters that may assist in determining the nature of these lesions, and histological exam is required for definitive diagnosis. Surgical excision is the treatment of choice. Post-surgical management includes thyrotropin suppression to prevent recurrence. Radioiodine ablation was not thought to be necessary in this case, as there was no evidence of remaining iodine-avid lesions in the spinal cord.

\section{References}

1. Cordes S, Nelson JJ. Papillary carcinoma arising in median ectopic thyroid tissue: Management of the thyroid gland. Ear Nose Throat J. 2010 May;89(5):E4-7.

2. Oh SW, Park DW, Kim SY et al. Primary follicular carcinoma arising in ectopic thyroid tissue of the lateral neck: A case report. J Korean Soc Radiol. 2010;63:413-7.

3. Cervera PFJ, Roquette GJ, Bartual PJ. Ectopic primitive thyroid papillary carcinoma: Report of a case and review of literature. Acta Otorhinolaryngol Esp. 1994;45:124-7.
4. Klubo-Gwiezdzinska J, Peter Manes R, Chia S et al. Ectopic cervical thyroid carcinoma: Review of the literature with illustrative case series. J Clin Endocrinol Metab. 2011 Sep;96(9): 2684-2691.

5. Hollander EJ, Visser MJ, van Baalen JM. Accessory thyroid gland at carotid bifurcation presenting as a carotid body tumor: Case report and review of the literature. J Vasc Surg. 2004;39:260-2.

6. Deda G, Tezic T, Karagol U et al. Ectopic thyroid tissue causing cord compression. Turkish Neurosurg. 1991;2:77-9.

7. Richmond L, Whittaker JS, Deirahiya AK et al. Intracardiac ectopic thyroid: A case report and review of published cases. Thorax. 1990;45(4):273-94.

8. Rubenfeld S, Joseph UA, Schwartz MR et al. Ectopic thyroid in the right carotid triangle. Arch Otolaryngol Head Neck Surg. 1988;114(8):913-915.

9. Ramos-Gabatin A, Pretorius HT. Radionuclide turnover studies on ectopic thyroid gland: Case report and survey of the literature. J Nuclear Med. 1985;26(3):258-62.

10. Hall TS, Caslowitz P, Caroline P et al. Substernal goiter versus intrathoracic aberrant thyroid: A critical difference. Ann Thorac Surg. 1988;46:684-5.

11. Karlic MR, Grillo H, Wang C. Substernal goiter: Analysis of 80 patients from Massachusetts General Hospital. Am J Neurosurg. 1985;149:283-7.

12. Osammor JY, Bulman $\mathrm{CH}$, Blewitt $\mathrm{RW}$ et al. Intralaryngotracheal thyroid. J Laryngol Otology. 1990;104:733-6.

13. Choi JY, Kim JH. A case of an ectopic thyroid gland at the lateral neck masquerading as a metastatic papillary thyroid carcinoma. J Korean Med Sci. 2008;23:548-50.

14. Mishriki YY, Lane BP, Lozowski MS et al. Hürthle cell tumor arising in the mediastinal ectopic thyroid and diagnosed by fine needle aspiration: Light microscopic and ultrastructural features. Acta Cytol. 1983;27:188-92.

15. Fumarola A, Trimboli P, Cavaliere R et al. Thyroid papillary carcinoma arising in ectopic thyroid tissue within a neck branchial cyst. World J Surg Oncol. 2006;4:24.

16. Shah B, Ravichand CS, Juluri $S$ et al. Ectopic thyroid cancer. Ann Thorac Cardiovasc Surg. 2007;13(2):122-4.

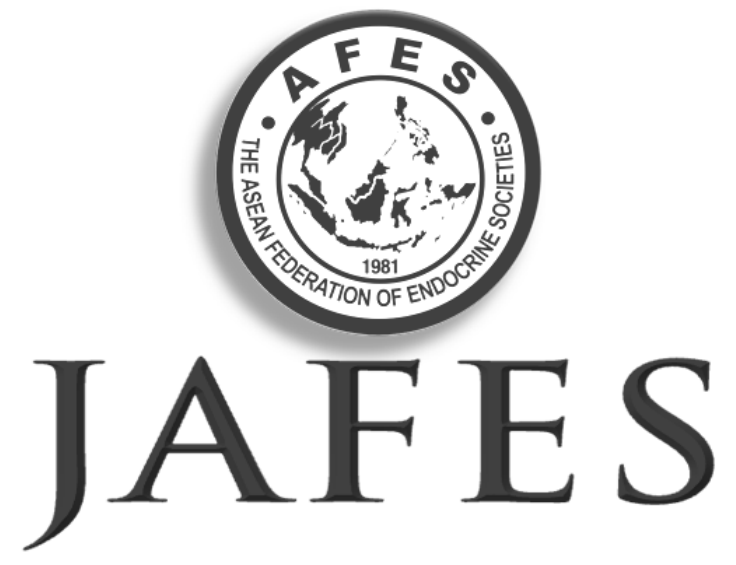

\section{Topics with cultural interest $=$ Images of Asia at JAFES asia.com}

